\title{
The Dependence of Electrical Resistivity-Saturation Relationships on Multiphase Flow Instability
}

\author{
Zoulin Liu and Stephen M. J. Moysey \\ Department of Environmental Engineering and Earth Science, Clemson University, Clemson, SC 29670, USA \\ Correspondence should be addressed to Stephen M. J. Moysey, smoysey@clemson.edu
}

Received 9 August 2012; Accepted 5 September 2012

Academic Editors: E. Liu and H. Perroud

Copyright ( $) 2012$ Z. Liu and S. M. J. Moysey. This is an open access article distributed under the Creative Commons Attribution License, which permits unrestricted use, distribution, and reproduction in any medium, provided the original work is properly cited.

\begin{abstract}
We investigate the relationship between apparent electrical resistivity and water saturation during unstable multiphase flow. We conducted experiments in a thin, two-dimensional tank packed with glass beads, where Nigrosine dyed water was injected uniformly along one edge to displace mineral oil. The resulting patterns of fluid saturation in the tank were captured on video using the light transmission method, while the apparent resistivity of the tank was continuously measured. Different experiments were performed by varying the water application rate and orientation of the tank to control the generalized Bond number, which describes the balance between viscous, capillary, and gravity forces that affect flow instability. We observed the resistivity index to gradually decrease as water saturation increases in the tank, but sharp drops occurred as individual fingers bridged the tank. The magnitude of this effect decreased as the displacement became increasingly unstable until a smooth transition occurred for highly unstable flows. By analyzing the dynamic data using Archie's law, we found that the apparent saturation exponent increases linearly between approximately 1 and 2 as a function of generalized Bond number, after which it remained constant for unstable flows with a generalized Bond number less than -0.106 .
\end{abstract}

\section{Introduction}

Multiphase fluid flow in porous media is an important problem for applications including petroleum production [1-3], migration of nonaqueous phase liquids (NAPLs) in soils and aquifers [4-6], and $\mathrm{CO}_{2}$ sequestration [7]. Viscous, capillary and gravity forces interact in immiscible two phase flow systems to produce stable or unstable flow regimes [811]. In a stable flow regime the displacement of one fluid for another will occur along a stable front. In unstable flow regimes, fingering can occur along the displacement front. As a result, the invading fluid phase can bypass significant amounts of the original fluid phase, leaving it in place in the medium.

Electrical resistivity measurements are commonly used to investigate fluid saturations in multiphase flow systems [1216]. The resistivity index provides an expression of resistivity for multiphase flow systems that is directly related to the degree of water saturation of the medium, $S_{w}$, through Archie's law [17]. When mineral surface conductivity is insignificant, the resistivity index $I_{R}$ is equal to the ratio of the resistivity of the sample $\left(\rho_{w}\right)$ measured at saturation $S_{w}$ to the resistivity of the sample measured at $100 \%$ water saturation $\left(\rho_{s}\right)$ (Equation (1)). The saturation exponent, $n$, is an empirical constant that is conceptually related to the connectivity of the electrically conductive phase, that is, water:

$$
I_{R}=\frac{\rho_{w}}{\rho_{s}}=S_{w}{ }^{-n}
$$

The saturation exponent is usually determined experimentally from measurements of $I_{R}$ and $S_{w}$ using (1). For example, Sweeney and Jennings [18] experimentally found the saturation exponent to be 1.61 for water wet carbonates, though this increased up to 12.27 for samples treated to become oil wet. Zhou et al. [16] used percolation models to show that the saturation exponent in strongly water wet materials is on the order of 1.9, whereas it can increase to over 3.5 for intermediate and oil wet systems. The saturation exponent is dependent on the presence of nonconductive 
fluid in the pore space and wettability of the rock [12, 19]. Although Archie's Law is widely used to determine fluid saturation from resistivity measurements, it is not always valid as the saturation-resistivity relationship depends on the wettability, saturation history, content of clay minerals, salinity of the brine phase and distribution of water and oil in the rock $[3,12,15,16]$. Most experimental studies of the dependence between saturation and apparent resistivity were based on the assumption that fluid distribution within the sample was homogeneous, which can be rarely obtained during a transient displacement experiment. We experimentally investigate the influence of flow instability on apparent resistivity and the saturation exponent in Archie's law. To this end, multiphase flow experiments are conducted where water is used to displace mineral oil in a two-dimensional (2D) flow system. In this paper, we conduct a series of experiments where the stability of the flow is controlled by varying the water inflow rate and angle of the tank. Measurements of the bulk resistivity of the tank are obtained during the flow experiments. Transient estimates of average water saturation in the tank, $S_{w}$ are derived from video collected using the light transmission method. Using these measurements we observe the relationship between saturation and resistivity for a range of flow conditions. We further evaluate whether there is a dependence of the saturation exponent in Archie's law on the flow conditions in a porous medium.

\section{Background on Flow Instability}

It is well known that variations in the magnitude and connectivity of permeability could lead to flow channeling in reservoirs and consequently to a reduction in oil production [20]. Even in a homogeneous medium, flow instability can cause viscous fingering that also increases the residual oil volume left behind in a reservoir $[8,9]$. Flow instability is affected by the cumulative effects of capillary, buoyancy, and viscous forces. For example, viscous forces can destabilize the displacement front into narrow fingers if a less viscous fluid displaces a more viscous fluid, whereas gravity plays a stabilizing effect when a lighter fluid is on top of a denser phase [10]. The balance between forces in a two phase flow system can be quantified using the dimensionless Bond and capillary numbers along with the viscosity ratio. The viscosity ratio $(M)$ is defined as the viscosity of displacing fluid $\mu_{w}$ divided by the viscosity of the displaced fluid $\mu_{n}$. Viscous fingering can be observed when the viscosity ratio is less than 1 and the viscous force overcomes capillary and gravity effects.

The Bond number $\left(B_{o}\right)$, given in (2), expresses the relative importance of gravitational to capillary forces in a multiphase flow system $[9,10]$. In contrast, the capillary number $\left(C_{a}\right)$ in $(3)$ expresses the balance between viscous to capillary forces $[9,10,21]$ :

$$
\begin{gathered}
B_{o}=\frac{\Delta P_{\text {grav }}}{\Delta P_{\text {cap }}}=\frac{\Delta \rho g a^{2}}{\gamma} \sin \varphi, \\
C_{a}=\frac{\Delta P_{\text {visc }}}{\Delta P_{\text {cap }}}=\frac{\mu_{w} \nu a^{2}}{\gamma k} .
\end{gathered}
$$

In these expressions, $\mu_{w}$ is the viscosity of wetting fluid, $v$ is the filtration or Darcy velocity, $a$ is the typical pore radius, $\gamma$ is surface tension, $\Delta \rho$ is the density difference between the two fluids, $g$ is the acceleration due to the gravity, $\varphi$ is the angle of flow relative to horizontal, and $k$ is the permeability of the porous medium $[9,10]$. The capillary and Bond numbers can be combined to produce the generalized Bond number $\left(B_{o}^{*}\right)$ given in the following $[9,10]$ :

$$
B_{o}^{*}=B_{o}-C_{a}=\frac{a^{2}}{\gamma k}\left(\Delta \rho g k \sin \varphi-\mu_{w} v\right) .
$$

The value of the generalized Bond number plays a critical role for determining the occurrence of viscous instabilities. For $B_{o}^{*}>0$ the flow is stable and a compact and flat displacement front occurs as illustrated in Figure 1(a). However, when $B_{o}^{*}<0$ the flow is unstable and fingering produces an uneven and often rapid movement of the infiltrating phase through the displaced phase in a porous medium (Figure 1(b); the white region in the images corresponds to oil, whereas the black areas correspond to Nigrosine dyed water). Despite the obvious contrast in macroscopic behavior, Méheust et al. note that a radical change in the local dynamics of the interface does not occur during the transition between stable and unstable displacement [10].

\section{Methods and Experimental Setup}

The main goal of this work is to determine the relationship between the saturation exponent in Archie's law and the degree of flow instability in a porous medium as quantified by the Bond and Capillary numbers. To achieve this objective, resistivity index curves were measured during the displacement of mineral oil by water in a $2 \mathrm{D}$ tank packed with glass beads. Four-wire resistance measurements were collected throughout the experiment while the light transmission method was used to simultaneously monitor changes in saturation. The effect of gravity on flow instability is controlled by changing the orientation of the tank to achieve different Bond numbers. Experiments at different flow rates were conducted to control the relative importance of viscous forces by varying the capillary number.

3.1. Experimental Setup. The fluids used in these experiments are water and mineral oil (EMD Chemicals, NJ, USA). The properties of each fluid are given in Table 1. We focus on a situation where a denser fluid with low viscosity (water) displaces a less dense, more viscous fluid (mineral oil) from below leading to a low viscosity ratio (0.015). Viscous fingering is therefore possible in this system. Negrosine (Acros Organics) dye was added to the water phase to provide contrast with the clear mineral oil to allow visual tracking of the displacement front and the development of fingers. This particular dye was selected because it did not partition from the water to oil phase in initial static tests conducted in beakers. The electrical conductivity of the water used in each experiment varied from $71.8-91.5 \mu \mathrm{s} / \mathrm{cm}$.

The experiments were conducted in the specially designed 2D acrylic tank shown in Figure 2. The dimensions 


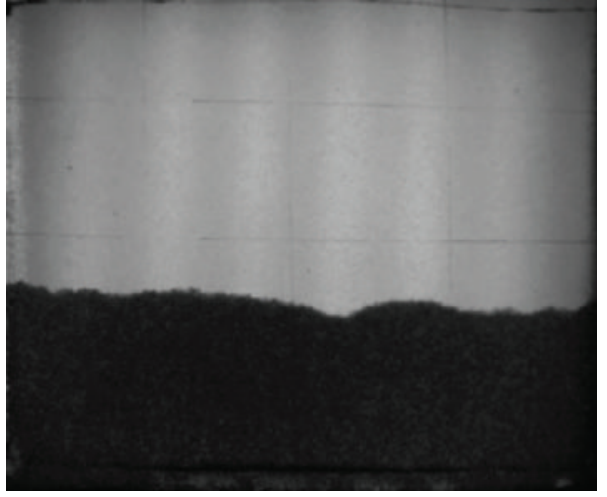

(a)

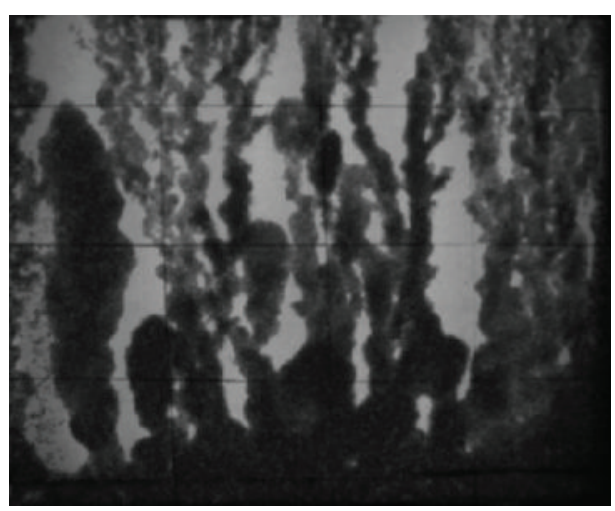

(b)

FIgURE 1: Comparison of (a) stable flow conditions $\left(B_{o}^{*}=0.00612\right)$ and $(\mathrm{b})$ unstable flow conditions $\left(B_{o}^{*}=-0.248\right.$; experiment \#12 in Table 3 ) for oil (white) displaced by Nigrosine dyed water (black).

TABle 1: Properties of the fluids used in the experiments.

\begin{tabular}{lc}
\hline \multicolumn{2}{c}{ Wetting phase, water (with $0.05 \mathrm{~g} / \mathrm{L}$ nigrosine) } \\
\hline Density, $\rho_{w}$ & $1000 \mathrm{~kg} / \mathrm{m}^{3}$ \\
Dynamic viscosity, $\mu_{w}$ & $1.002 E-3 \mathrm{~N} . \mathrm{s} / \mathrm{m}^{2}$ \\
\hline \multicolumn{2}{c}{ Non-wetting phase, mineral oil } \\
\hline Density, $\rho_{n}$ & $880 \mathrm{~kg} / \mathrm{m}^{3}$ \\
Dynamic viscosity, $\mu_{n}$ & $0.068 \mathrm{~N} . \mathrm{s} / \mathrm{m}^{2}$ \\
Interfacial tension $[22], \gamma$ & $0.049 \mathrm{~N} / \mathrm{m}^{2}$ \\
\hline Viscosity Ratio, $M$ & 0.015 \\
\hline
\end{tabular}

TABLE 2: Physical properties of the flow system.

\begin{tabular}{lc}
\hline Length & $40 \mathrm{~cm}$ \\
width & $45 \mathrm{~cm}$ \\
Thickness & $1.25 \mathrm{~cm}$ \\
Porosity, $\varepsilon$ & 0.30 \\
Formation factor, $F_{f}$ & 3.04 \\
Permeability, $k$ & 57 Darcy \\
Grain Size, $D$ & $2 \mathrm{~mm}$ \\
\hline
\end{tabular}

of the interior flow cell of the tank are $45 \mathrm{~cm} \times 40 \mathrm{~cm} \times$ $1.25 \mathrm{~cm}$. For all of the experiments in the study, the flow cell was packed with $2 \mathrm{~mm}$ diameter glass beads (Walter Stern). The entire cell was designed to be pressure sealed, thereby allowing for the tank to be oriented at arbitrary geometries. The outlet pressure of the tank was held at a constant positive pressure by keeping the discharge reservoir above the tank (Figure 2). Details regarding the physical properties of the tank are summarized in Table 2.

The tank could tilt to arbitrary angles so as to vary the effect of gravity on flow and control the Bond number. The component of gravity acting on the flow system is determined by $g_{\varphi}=g \times \sin (\varphi)$, where $g$ is acceleration due to gravity and $\varphi$ is the angle of the tank relative to horizontal. For each experiment water was injected into the tank at a constant rate selected to achieve a specified capillary number using a variable rate peristaltic pump (pump head: HV-07015-20, Master Flex). The displacing water phase is

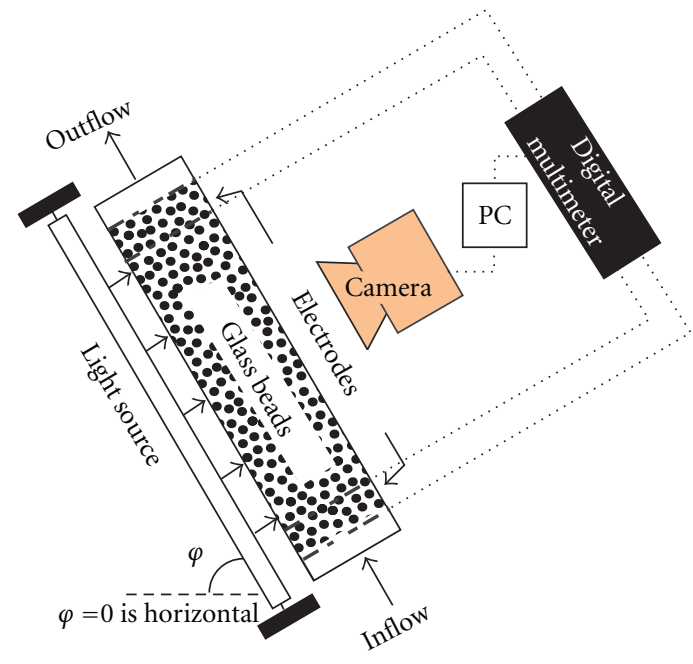

FIGURE 2: Side view sketch of the experimental setup for the resistivity cell with light transmission imaging and resistivity measurement systems.

injected through a porous plastic plate covering the entire inlet surface of the beads to ensure the injection is uniform. The displaced oil phase is expelled from a similar outlet port at the opposite end of the flow cell. Both gravity effects, that is, Bond number, and flow rate, that is, capillary number, influence the stability of flow in our experiments and can be changed independently of each other. A complete listing of tank orientations and flow rates used in the experiments is given in Table 3.

The bulk DC resistance of the tank was determined using the four-electrode method [4, 24]. Two pieces of copper mesh were anchored across the inflow and outflow sides of the tank to act as current electrodes. Two additional copper strips were positioned $2 \mathrm{~cm}$ away from each potential electrode within the tank to act as potential electrodes (Figure 2). A National Instruments PXI system with 7.5-digit digital multimeter and multiconfiguration matrix module (NI PXI-4071, PXI-2530) were used to measure the DC 
TABle 3: Summary of the tank inclination angle $(\varphi)$, pumping rate $(Q)$, and fluid conductivity $\left(\sigma_{w}\right)$ for each of the 34 experiments. The corresponding characteristic numbers $C_{a}, B_{o}$ and $B_{o}^{*}$ are also given (pore size estimated as $0.414 \times$ grain size following [23]).

\begin{tabular}{|c|c|c|c|c|c|c|c|}
\hline Experiment index & 1 & 2 & 3 & 4 & 5 & 6 & 7 \\
\hline$Q(\mathrm{~mL} / \mathrm{min})$ & 189 & 251 & 67 & 119 & 157 & 27 & 67 \\
\hline$\varphi$ (degree) & 90 & 90 & 90 & 90 & 90 & 90 & 90 \\
\hline$\sigma_{w}(\mu \mathrm{s} / \mathrm{cm})$ & 98 & 93 & 90.2 & 93.7 & 81.4 & 126 & 94.7 \\
\hline$B_{o}$ & $1.65 E-02$ & $1.65 E-02$ & $1.65 E-02$ & $1.65 E-02$ & $1.65 E-02$ & $1.65 E-02$ & $1.65 E-02$ \\
\hline$C_{a}$ & $1.39 E-01$ & $1.85 E-01$ & $4.94 E-02$ & $8.78 E-02$ & $1.16 E-01$ & $1.99 E-02$ & $4.94 E-02$ \\
\hline$B_{o}^{*}$ & $-1.23 E-01$ & $-1.69 E-01$ & $-3.30 E-02$ & $-7.13 E-02$ & $-9.94 E-02$ & $-3.47 E-03$ & $-3.30 E-02$ \\
\hline Experiment index & 8 & 9 & 10 & 11 & 12 & 13 & 14 \\
\hline$Q(\mathrm{~mL} / \mathrm{min})$ & 119 & 27 & 189 & 251 & 358 & 67 & 99 \\
\hline$\varphi$ (degree) & 90 & 90 & 90 & 90 & 90 & 90 & 90 \\
\hline$\sigma_{w}(\mu \mathrm{s} / \mathrm{cm})$ & 82 & 78.7 & 79.5 & 76.7 & 78.4 & 80.1 & 83.1 \\
\hline$B_{o}$ & $1.65 E-02$ & $1.65 E-02$ & $1.65 E-02$ & $1.65 E-02$ & $1.65 E-02$ & $1.65 E-02$ & $1.65 E-02$ \\
\hline$C_{a}$ & $8.78 E-02$ & $1.99 E-02$ & $1.39 E-01$ & $1.85 E-01$ & $2.64 E-01$ & $4.94 E-02$ & $7.30 E-02$ \\
\hline$B_{o}^{*}$ & $-7.13 E-02$ & $-3.47 E-03$ & $-1.23 E-01$ & $-1.69 E-01$ & $-2.48 E-01$ & $-3.30 E-02$ & $-5.66 E-02$ \\
\hline Experiment index & 15 & 16 & 17 & 18 & 19 & 20 & 21 \\
\hline$Q(\mathrm{~mL} / \mathrm{min})$ & 89 & 52 & 146 & 119 & 67 & 27 & 67 \\
\hline$\varphi$ (degree) & 90 & 90 & 90 & 90 & 90 & 90 & 90 \\
\hline$\sigma_{w}(\mu \mathrm{s} / \mathrm{cm})$ & 74.8 & 77.6 & 74.2 & 79 & 77.6 & 78.3 & 87 \\
\hline$B_{o}$ & $1.65 E-02$ & $1.65 E-02$ & $1.65 E-02$ & $1.65 E-02$ & $1.65 E-02$ & $1.65 E-02$ & $1.65 E-02$ \\
\hline$C_{a}$ & $6.57 E-02$ & $3.84 E-02$ & $1.08 E-01$ & $8.78 E-02$ & $4.94 E-02$ & $1.99 E-02$ & $4.94 E-02$ \\
\hline$B_{o}^{*}$ & $-4.92 E-02$ & $-2.19 E-02$ & $-9.13 E-02$ & $-7.13 E-02$ & $-3.30 E-02$ & $-3.47 E-03$ & $-3.30 E-02$ \\
\hline Experiment index & 22 & 23 & 24 & 25 & 26 & 27 & 28 \\
\hline$Q(\mathrm{~mL} / \mathrm{min})$ & 189 & 251 & 67 & 67 & 67 & 67 & 67 \\
\hline$\varphi$ (degree) & 90 & 90 & 30 & 30 & 30 & 0 & 45 \\
\hline$\sigma_{w}(\mu \mathrm{s} / \mathrm{cm})$ & 71.8 & 72.8 & 78 & 91.5 & 90 & 81.1 & 80.5 \\
\hline$B_{o}$ & $1.65 E-02$ & $1.65 E-02$ & $8.23 E-03$ & $8.23 E-03$ & $8.23 E-03$ & $0.00 E+00$ & $1.16 E-02$ \\
\hline$C_{a}$ & $1.39 E-01$ & $1.85 E-01$ & $4.94 E-02$ & $4.94 E-02$ & $4.94 E-02$ & $4.94 E-02$ & $4.94 E-02$ \\
\hline$B_{o}^{*}$ & $-1.23 E-01$ & $-1.69 E-01$ & $-4.12 E-02$ & $-4.12 E-02$ & $-4.12 E-02$ & $-4.94 E-02$ & $-3.78 E-02$ \\
\hline Experiment index & 29 & 30 & 31 & 32 & 33 & 34 & \\
\hline$Q(\mathrm{~mL} / \mathrm{min})$ & 67 & 67 & 67 & 67 & 358 & 358 & \\
\hline$\varphi$ (degree) & 15 & 60 & 60 & 60 & 90 & 90 & \\
\hline$\sigma_{w}(\mu \mathrm{s} / \mathrm{cm})$ & 82.8 & 84 & 79.2 & 84.7 & 89.1 & 88.5 & \\
\hline$B_{o}$ & $4.26 E-03$ & $1.42 E-02$ & $1.42 E-02$ & $1.42 E-02$ & $1.65 E-02$ & $1.65 E-02$ & \\
\hline$C_{a}$ & $4.94 E-02$ & $4.94 E-02$ & $4.94 E-02$ & $4.94 E-02$ & $2.64 E-01$ & $2.64 E-01$ & \\
\hline$B_{o}^{*}$ & $-4.52 E-02$ & $-3.52 E-02$ & $-3.52 E-02$ & $-3.52 E-02$ & $-2.48 E-01$ & $-2.48 E-01$ & \\
\hline
\end{tabular}

resistivity of the tank while switching the polarity of the current electrodes to avoid electrode polarization effects. Prior to running the flow experiments, the flow cell was filled with a saline solution and measurements were taken to calibrate the geometric factor relating tank bulk resistance to resistivity. After packing the tank with the glass beads the formation factor in Archie's law was determined to be 3.04 for our experiments by measuring resistance for several different solution conductivities. Surface conductivity effects for these large glass beads are assumed to be negligible given insignificant imaginary conductivity responses measured by spectral induced polarization [25].

The system developed for the light transmission measurements $[6,26,27]$ contains a light source and detector (Figure 2). In this experiment a scientific digital camera (DFK 41BU02.H USB CCD, Imaging Source) with a $5 \mathrm{~mm}$ lens (H0514-MP, Imaging Source) is used as the detector to quantify the intensity of light transmitted through the tank. This camera has resolution of $1280 \times 960$ pixels for 32 bit images, which provides a spatial resolution of $0.39 \mathrm{~mm}$ per pixel or about 2 pixels per pore for a distance between the camera and the tank of $40 \mathrm{~cm}$. The camera is controlled by a host computer using a LabView (National Instruments) program to obtain images at a specific frame rate during the fluid displacement. The pictures that the camera takes are in raw bmp format with no compression. Images are later converted into gray scale and analyzed using MATLAB. The light transmitted through the tank is generated by an array of fluorescent bulbs ( $13 \mathrm{~W}$ each, Bi-Pin, MA) mounted to the back of the tank in a manner allowing it to move with the tank when the experimental angle is changed.

The background reference image obtained before water is injected is subtracted from each subsequent image to overcome problems related to variations in light intensity 


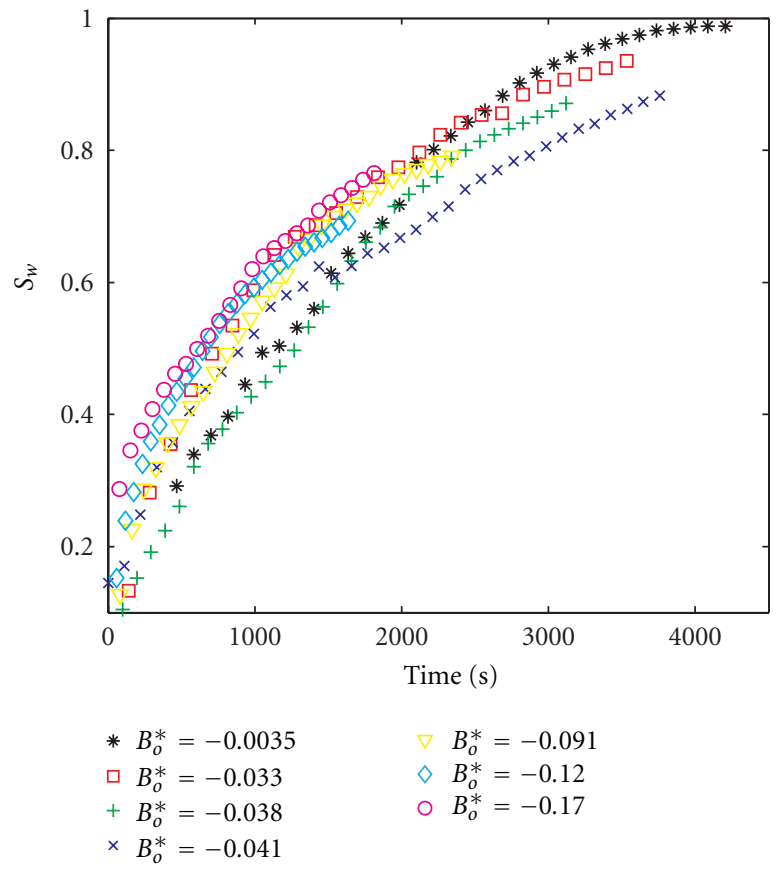

(a)

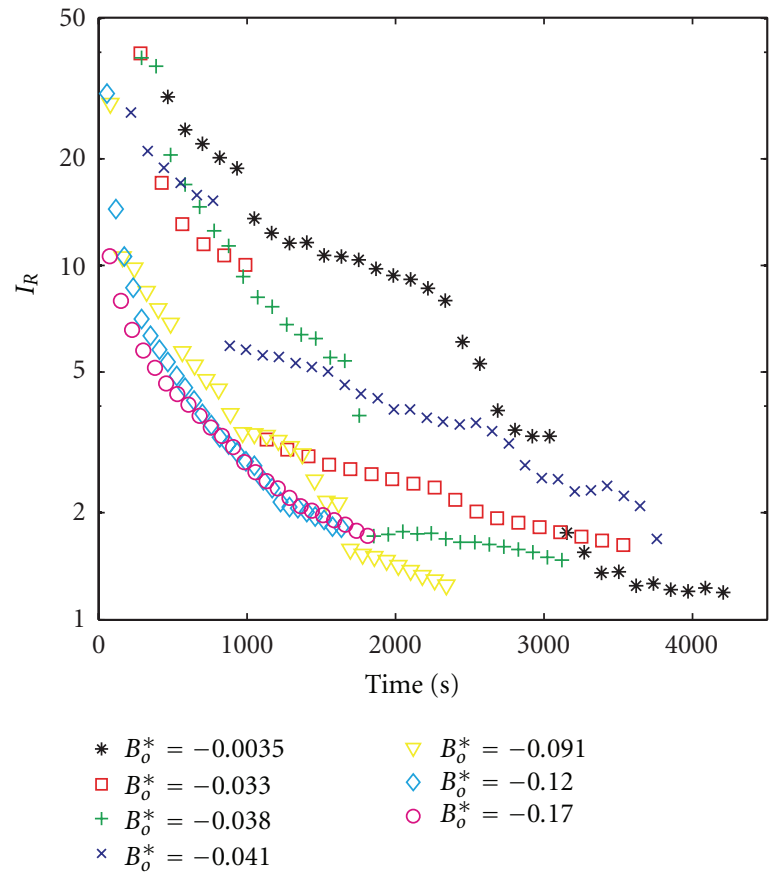

(b)

FIGURE 3: Changes in (a) average tank saturation and (b) resistivity index through time for varying values of $B_{o}^{*}$ (data shown for experiment index $=9,7,28,25,17,10,11)$.

due to the specific arrangement of light bulbs in the array. The intensity $\left(I_{0}-I\right)$ of the corrected image was found to have a linear relationship with the water saturation $S_{w}$ inside the porous medium as shown in the following:

$$
S_{w}=\frac{\left[1.0759 \times\left(I_{0}-I\right)-3.3557\right]}{100} .
$$

This equation is obtained from calibration experiments using a small chamber with the same material, thickness, and packing of glass beads to obtain a porosity of about 0.30 , consistent with the flow cell.

With the experimental setup described above, 2 main series of 34 experiments are conducted: one series is at constant Bond number of 0.0165 and the other is at constant capillary number of 0.0494 . Table 3 summarizes the experimental conditions for all experiments in terms of the orientation of the tank $(\varphi)$, pumping rates $(Q)$, water conductivity $(\sigma)$, and the corresponding Capillary number $\left(C_{a}\right)$, Bond number $\left(B_{o}\right)$, and generalized Bond number $B_{o}^{*}$.

\section{Results}

The range of the Bond numbers that can be achieved by rotating the tank, that is, 0 to 0.0165 , is smaller than the range of capillary numbers that can be achieved by changing the flow rate, that is, 0 to 0.264 . Therefore, we can obtain the largest range of generalized Bond numbers by changing flow rate. The maximum generalized Bond number used in the experiments is -0.0035 because the digital multimeter was not able to read the high resistivity of the mineral oil in completely stable situations where water uniformly displaced the oil. The lowest (i.e., most negative) generalized Bond number investigated is -0.248 as the medium tended to compact under high internal pressures if higher flow rates were applied in the closed cell.

4.1. Saturation and Resistivity Index. The average water saturation and resistivity index of the tank over time are shown in Figure 3 for different values of the generalized Bond number. Saturations change relatively smoothly in most cases as water displaces the oil. Differences between the curves are apparent, but trends for different generalized Bond numbers are not clear. In contrast, the resistivity index curves show a distinctive change in behavior with generalized Bond number. For small values of $B_{o}^{*}$, that is, values near zero where flow is more stable, the resistivity index curves show large, sudden drops. In contrast, for large negative values of $B_{0}^{*}$, in which case the flow is highly unstable with many thin fingers formed, the reduction in resistivity index over time is smooth and regular. This result is indicative of the high sensitivity of resistivity measurements to the geometry of the water phase in the medium. Note that we use resistivity index here since the fluid resistivity varied between some of the experiments (Table 3 ).

At small negative values of $B_{o}^{*}$ the flow is stable and the water advances either as a uniform front or as large, individual fingers. Using the video collected during the experiment, each jump in resistivity index can be correlated with the time at which a finger of water reaches the tank's upper current electrode, thereby completing a new pathway 

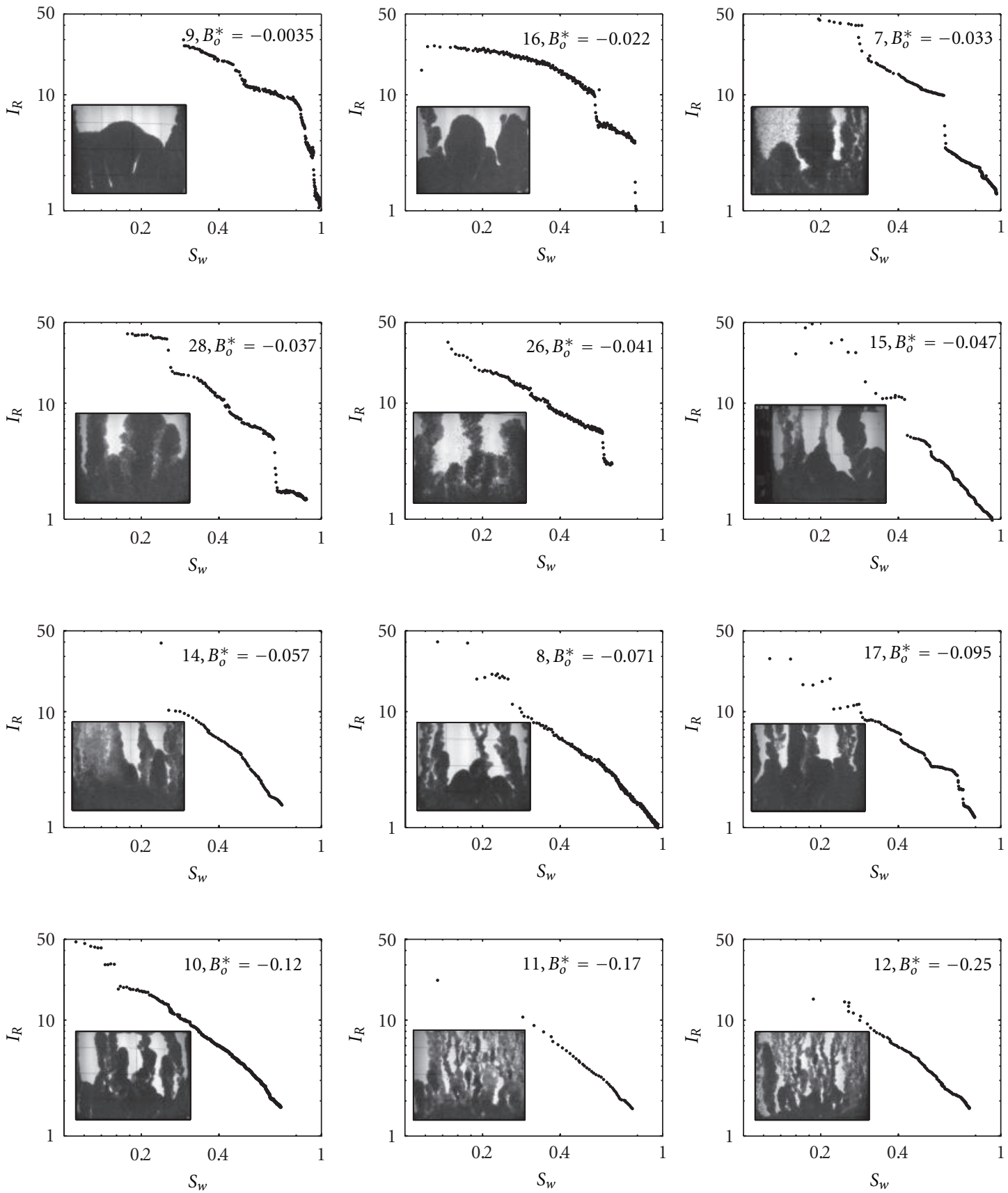

Figure 4: Dependence of resistivity index on saturation for different values of $B_{o}^{*}$ (the experimental index and $B_{o}^{*}$ are given in each figure). A representative image of the flow conditions during each experiment is shown with the inset picture.

for current to flow through the medium. At large negative values of $B_{o}^{*}$ the flow is highly unstable, producing many thin fingers. The fingers tend to reach the tank outflow in a more uniform manner, producing the relatively smooth change in resistivity observed in Figure 3(b). The patterns of fingering observed in our experiments (Figure 4), that is, increasing number of fingers and decreasing finger thickness with increasing generalized Bond number, is consistent with observations from experiments by Løvoll et al. [9] and Méheust et al. [10] though these authors did not measure resistivity.
Based on experimental data, Méheust et al. [10] suggest a power law with an exponent of -0.55 to relate the front width of the fingering and the generalized Bond number:

$$
W=B_{o}^{*-0.55}
$$

where $W$ is the measured front width of the finger, that is, the root mean square maximum extension perpendicular to the flow direction. Therefore bigger discrete drops in the resistivity index at small values of the generalized Bond number can be attributed to wider fingers reaching the 


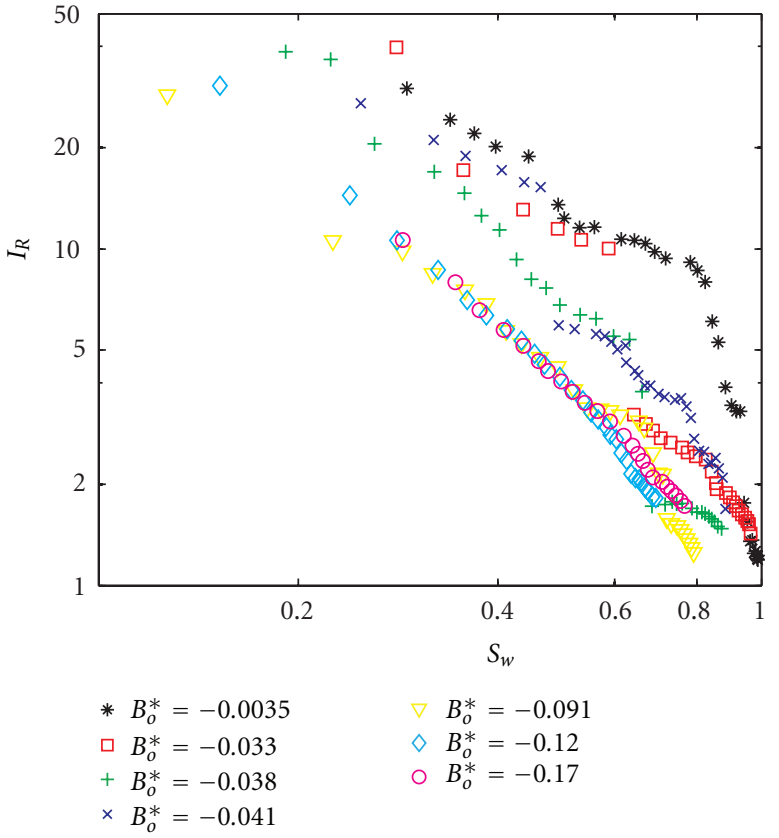

FIGURE 5: Direct comparison of resistivity index changes with saturation for different generalized Bond numbers.

electrodes and causing a larger portion of the flow cell to connect the electrodes. As the flow grows increasingly unstable, the conductive fingers have a smaller width and are more uniformly distributed in the tank, though individual fingers may form at different times. As a result, the resistivity change caused by an individual finger is small and the observed change in resistivity is smoother as a result of the progressive arrival of individual conductive fingers.

Figures 4 and 5 show the resistivity index versus water saturation for a subset of the generalized Bond numbers used in the experiments. For a given saturation value the resistivity index is generally lowest for unstable flow scenarios, that is, in Figure 5 the value of the generalized Bond number of the curves decreases (becomes more negative) from the top curve to the bottom. The slope of the resistivity index curves is flattest for small negative values of the generalized Bond number with changes in resistivity occurring as sharp drops when individual fingers reach the outflow end of the tank. As discussed previously, the magnitude of these drops in the resistivity index decrease as the generalized Bond number becomes more negative and the drops disappear when the flow is very unstable at $B_{o}^{*}=-0.123$ (Figure 5).

It is notable that the resistivity index versus saturation curves for $B_{o}^{*}=-0.123$ (Figure 5, diamond) and $B_{o}^{*}=$ -0.169 (Figure 5 , circle) overlap with each other, suggesting that these two unstable flow scenarios have a similar electrical behavior. In contrast, at higher generalized Bond numbers, for example, $B_{o}^{*}=-0.00347$, the flow is more stable and a very sharp drop in $I_{R}$ is observed at the end of the experiment. This rapid change in resistivity index occurs because the front of the water moving to the end the tank is relatively flat (i.e., lacks fingers) compared to the other experiments. In this case, both the water saturation and

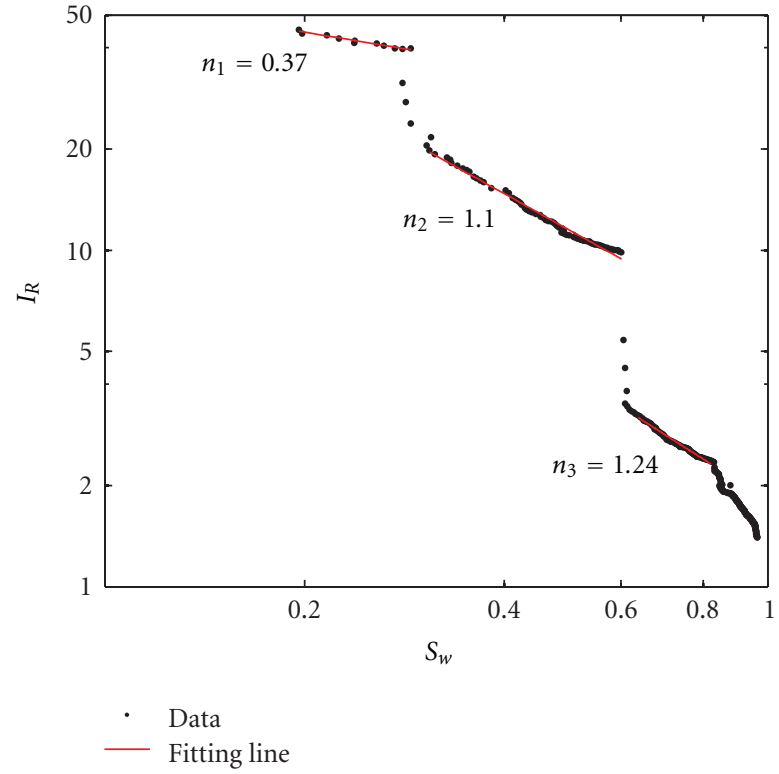

FIGURE 6: Illustration of how the saturation exponent was determined for experiments with discrete resistivity drops (data shown for experiment index 7).

resistivity index are approximately equal to 1 at the end of the experiment, indicating that the porous medium is almost fully saturated with water (i.e., high sweep efficiency) and that the resistivity is approximately equal that observed for the $100 \%$ water saturated tank.

4.2. Influence of Flow Instability on the Saturation Exponent. Archie's law (equation (1)) is typically assumed to apply to situations where fluids are distributed uniformly throughout a porous medium. We feel it is also valuable, however, to investigate how flow instability could affect the apparent properties of the formation during unstable flow. In Archie's law the logarithms of the resistivity index $\left(I_{R}\right)$ and saturation are linearly related with a slope equivalent to the negative of the saturation exponent $(n)$. We analyze the saturation and resistivity data obtained here in a similar way to obtain an apparent saturation exponent for each experiment. For some experiments, however, the slope of these curves varies as a function of saturation. This effect is at least partially a result of the resistivity drops discussed earlier, which are a consequence of the fact that the measurements represent a dynamic flow system where preferential flows are established within the spatially finite bounds of the tank. The saturation exponent was therefore estimated for distinct sections of the resistivity-saturation curve, ignoring the sudden drops in resistivity caused by the breakthrough of individual fingers of water (Figure 6). We acknowledge that the value of the apparent saturation exponent obtained in this way may not have the same physical meaning as in typical applications of Archie's law. Regardless, this approach still provides a way to summarize the experimental data in a way that allows for effective comparison between the experiments. 
TABLE 4: Saturation exponent determined for each experiment.

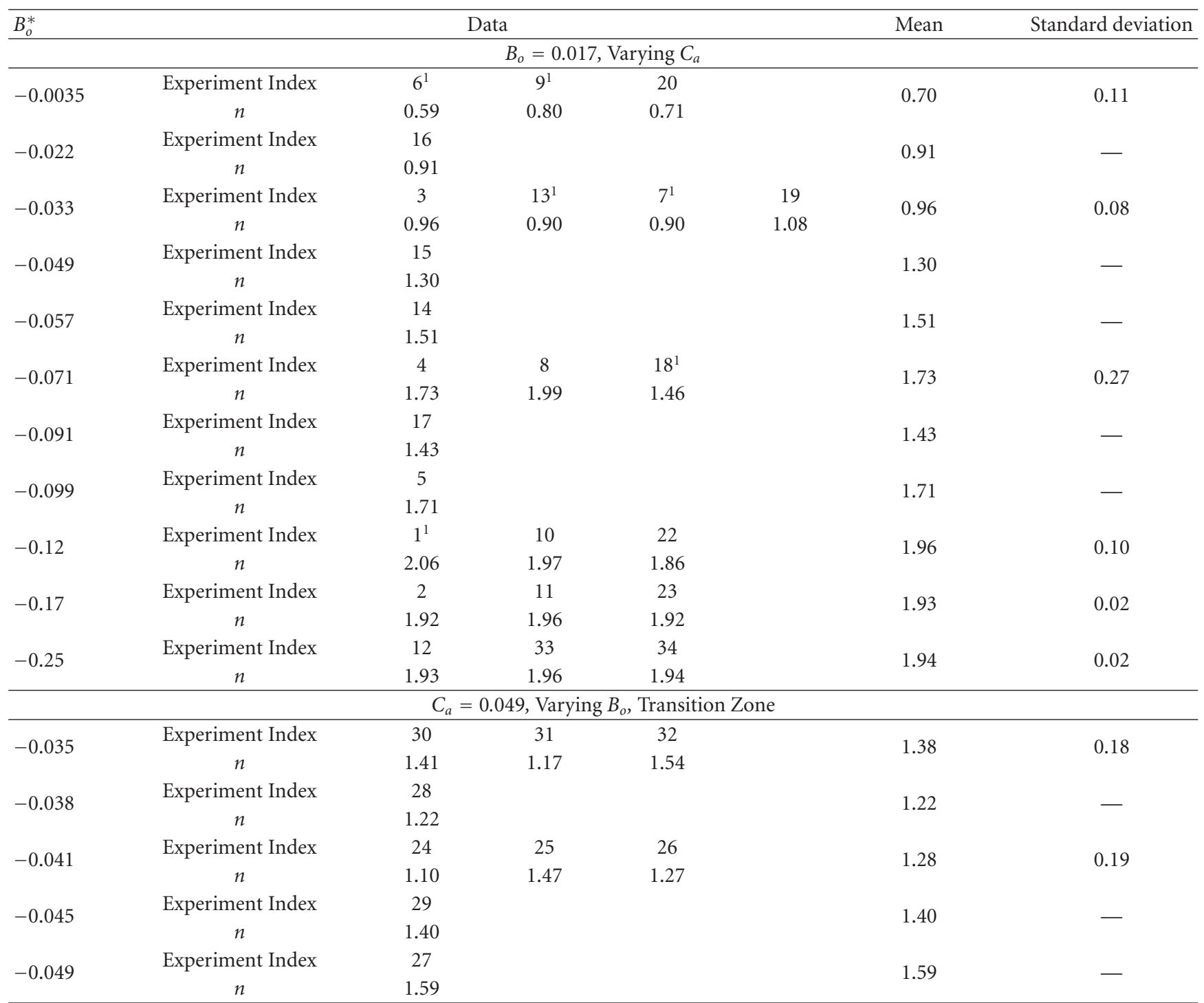

${ }^{1}$ Significant resistivity drops observed in the data.

Table 4 shows the apparent saturation exponent estimated for each experiment, averaging sections of the curves for the experiments found to have large, sudden resistivity drops. We take the average of these values to estimate the saturation exponent corresponding to a given generalized Bond number. For the unstable flow experiments, that is, large negative values of $B_{o}^{*}$, the resistivity change is continuous so the saturation exponent is estimated by fitting the data with a single slope. We can therefore evaluate how the apparent saturation exponent in our experiments varies as a function of generalized Bond number using the measured resistivity index versus water saturation curves (i.e., Figures 4 and 5).

We find that the apparent saturation exponent increases when flow becomes increasingly unstable, that is, $B_{o}^{*}$ becomes more negative (Figure 7). The saturation exponent reaches a constant value of 1.94 when generalized Bond number is less than -0.106 ; notably, this is consistent with the saturation index estimated by Zhou et al. [16] for strongly water wet materials using percolation theory. We quantify the relationship observed in Figure 7 between generalized Bond number and the saturation exponent as follows:

$$
\begin{gathered}
n=-9.3 \times B_{o}^{*}+0.9 \text { for }-0.106<B_{o}^{*}<-0.00347 \\
n=1.94 \text { for } B_{o}^{*}<-0.106
\end{gathered}
$$

The observed dependence of the saturation exponent on generalized Bond number demonstrates that relationships used to estimate fluid saturation from resistivity measurements, for example, Archie's law, must be dynamic and take into account the way in which a reservoir is managed and produced. The saturation exponent is fundamentally related to the geometry of the conductive and nonconductive phases within a porous medium, specifically the connectivity of the conductive phase. Given that instability has an overwhelming influence on fluid distributions during multiphase flow, this process will also strongly influence the saturation index. 


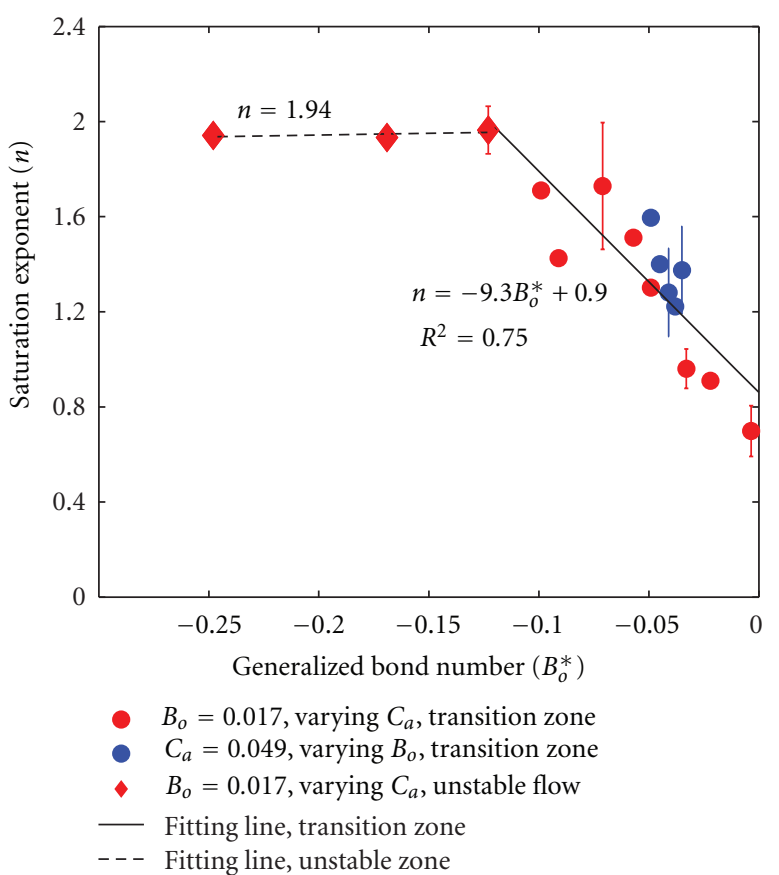

Figure 7: Saturation exponent as a function of generalized bond number $B_{o}^{*}$ for all experiments with a linear fit over the range $-0.106<B_{o}^{*}<-0.00347$. Error bars indicate the standard deviation in the saturation exponent estimate from experiments performed with the same value of $B_{o}^{*}$.

We identify the increasing connectivity of the water phase between the current electrodes as the primary cause for dependence of the saturation exponent on the generalized Bond number for $-0.106<B_{o}^{*}<-0.00347$. However, when the system reaches a certain minimum degree of connectivity due to flow instability, that is, when $B_{o}^{*}<-0.106$, there no longer appears to be a dependence between the saturation exponent and generalized Bond number. These results suggest that geophysicists must collaborate with petroleum engineers to understand the dynamics of a given flow system before attempting to estimate saturation from resistivity measurements.

\section{Conclusions}

The influence of flow instability on electrical resistivity measurements was investigated by displacing a light, but viscous mineral oil by water in a homogeneous porous medium. The experimental setup allowed the effects of gravity and flow rate to be controlled, thereby permitting the generalized Bond number to be changed between different experiments. Video showing the distribution of the fluid phases in the tank allowed us to determine the overall tank saturation throughout the experiment, while continuous measurements of average tank resistivity were also collected throughout each experiment.

In the resulting data we observed a transition between stable and unstable displacement. By analyzing the saturation and resistivity data using Archie's law, we found that the resulting apparent saturation exponent depends linearly on the generalized Bond number until it reaches a maximum of 1.94 for highly unstable flow systems, that is, when $B_{o}^{*}<$ -0.106 . These results suggest that the interpretation of fluid saturation from electrical resistivity measurements must take into account flow conditions within the subsurface, since the onset of flow instability is a primary control on the spatial distribution of the fluids in the subsurface.

\section{Acknowledgment}

Acknowledgment is made to the Donors of the American Chemical Society Petroleum Research Fund for support (or partial support) of this research.

\section{References}

[1] K. Mogensen, E. H. Stenby, and D. Zhou, "Studies of waterflooding in low-permeable chalk by use of X-ray CT scanning," Journal of Petroleum Science and Engineering, vol. 32, no. 1, pp. 1-10, 2001.

[2] D. Tiab and E. C. Donaldson, Petrophysics - Theory and Practice of Measuring Reservoir Rock and Fluid Transport Properties, Gulf Professional, 2nd edition, 2004.

[3] E. Toumelin and C. Torres-Verdín, "Object-oriented approach for the pore-scale simulation of DC electrical conductivity of two-phase saturated porous media," Geophysics, vol. 73, no. 2, pp. E67-E79, 2008.

[4] C. Aggelopoulos, P. Klepetsanis, M. A. Theodoropoulou, K. Pomoni, and C. D. Tsakiroglou, "Large-scale effects on resistivity index of porous media," Journal of Contaminant Hydrology, vol. 77, no. 4, pp. 299-323, 2005.

[5] T. W. J. Bauters, D. A. DiCarlo, T. S. Steenhuis, and J. Y. Parlange, "Preferential flow in water-repellent sands," Soil Science Society of America Journal, vol. 62, no. 5, pp. 11851190, 1998.

[6] N. Weisbrod, M. R. Niemet, and J. S. Selker, "Imbibition of saline solutions into dry and prewetted porous media," Advances in Water Resources, vol. 25, no. 7, pp. 841-855, 2002.

[7] N. B. Christensen, D. Sherlock, and K. Dodds, "Monitoring $\mathrm{CO}_{2}$ injection with cross-hole electrical resistivity tomography," Exploration Geophysics, vol. 37, pp. 44-49, 2006.

[8] G. M. Homsy, "Viscous fingering in porous-media," Annual Review of Fluid Mechanics, vol. 19, pp. 271-311, 1987.

[9] G. Løvoll, Y. Méheust, K. J. Måløy, E. Aker, and J. Schmittbuhl, "Competition of gravity, capillary and viscous forces during drainage in a two-dimensional porous medium, a pore scale study," Energy, vol. 30, no. 6, pp. 861-872, 2005.

[10] Y. Méheust, G. Loøvoll, K. J. Måløy, and J. Schmittbuhl, "Interface scaling in a two-dimensional porous medium under combined viscous, gravity, and capillary effects," Physical Review E, vol. 66, no. 5, Article ID 051603, 12 pages, 2002.

[11] J. P. Stokes, D. A. Weitz, J. P. Gollub et al., "Interfacial stability of immiscible displacement in a porous medium," Physical Review Letters, vol. 57, no. 14, pp. 1718-1721, 1986.

[12] W. G. Anderson, "Wettability literature survey-part 3: the effect of wettability on the electrical properties of porous media," Journal of Petroleum Technology, vol. 39, no. 13, pp. 1371-1378, 1986.

[13] S. Bekri, J. Howard, J. Muller, and P. M. Adler, "Electrical resistivity index in multiphase flow through porous media," Transport in Porous Media, vol. 51, no. 1, pp. 41-65, 2003. 
[14] M. J. Blunt, M. D. Jackson, M. Piri, and P. H. Valvatne, "Detailed physics, predictive capabilities and macroscopic consequences for pore-network models of multiphase flow," Advances in Water Resources, vol. 25, no. 8-12, pp. 1069-1089, 2002.

[15] A. K. Moss, X. D. Jing, and J. S. Archer, "Wettability of reservoir rock and fluid systems from complex resistivity measurements," Journal of Petroleum Science and Engineering, vol. 33, no. 1-3, pp. 75-85, 2002.

[16] D. Zhou, S. Arbabi, and E. H. Stenby, "A percolation study of wettability effect on the electrical properties of reservoir rocks," Transport in Porous Media, vol. 29, no. 1, pp. 85-98, 1997.

[17] G. E. Archie, "The electrical resistivity log as an aid in determining some reservoir characteristics," Petroleum Transactions of AIME, vol. 146, pp. 54-62, 1942.

[18] S. A. Sweeney and H. Y. Jennings, "Effect of wettability on the electrical resistivity of carbonate rock from a petroleum reservoir," Journal of Physical Chemistry, vol. 64, no. 5, pp. 551-553, 1960.

[19] D. Abdassah, P. Permadi, Y. Sumantri, and R. Sumantri, "Saturation exponent at various wetting condition: fractal modeling of thin-sections," Journal of Petroleum Science and Engineering, vol. 20, no. 3-4, pp. 147-154, 1998.

[20] J. M. Hovadik and D. K. Larue, "Static characterizations of reservoirs: refining the concepts of connectivity and continuity," Petroleum Geoscience, vol. 13, no. 3, pp. 195-211, 2007.

[21] R. Lenormand, "Liquids in porous media," Journal of Physics, vol. 2, pp. SA79-SA88, 1990.

[22] H. Yoon, M. Oostrom, and C. J. Werth, "Estimation of interfacial tension between organic liquid mixtures and water," Environmental Science and Technology, vol. 43, no. 20, pp. 7754-7761, 2009.

[23] J. F. Villaume, "Investigations at sites contaminated with dense, non-aqueous phase liquids (NAPLs)," Ground Water Monitoring Review, vol. 5, no. 2, pp. 60-74, 1985.

[24] G. F. Tagg, "Practical investigations of the earth resistivity method of geophysical surveying," Proceedings of the Physical Society, vol. 43, no. 3, pp. 305-323, 1931.

[25] N. Hao, J. Waterman, T. A. Kendall, S. M. Moysey, and D. Ntarlagiannis, "Resolving IP mechanisms using micron-scale surface conductivity measurements and column SIP data," Geochimica et Cosmochimica Acta, vol. 74, pp. A380-A380, 2010.

[26] C. J. G. Darnault, J. A. Throop, D. A. Dicarlo, A. Rimmer, T. S. Steenhuis, and J. Y. Parlange, "Visualization by light transmission of oil and water contents in transient two-phase flow fields," Journal of Contaminant Hydrology, vol. 31, no. 3-4, pp. 337-348, 1998.

[27] M. R. Niemet and J. S. Selker, "A new method for quantification of liquid saturation in $2 \mathrm{D}$ translucent porous media systems using light transmission," Advances in Water Resources, vol. 24, no. 6, pp. 651-666, 2001. 

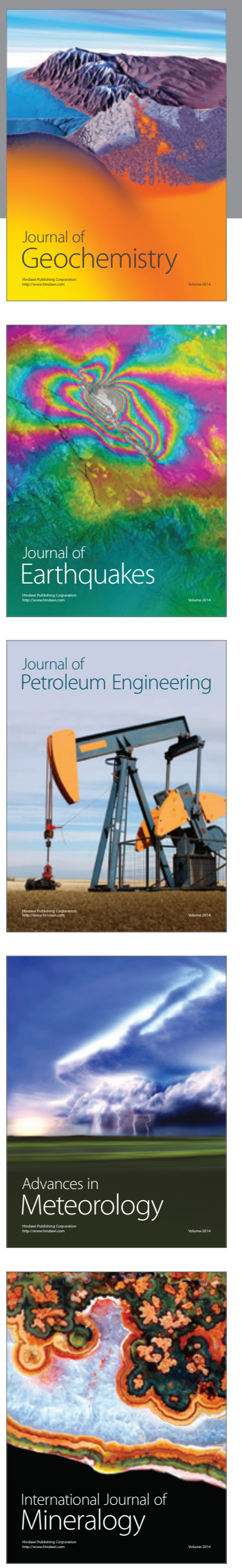
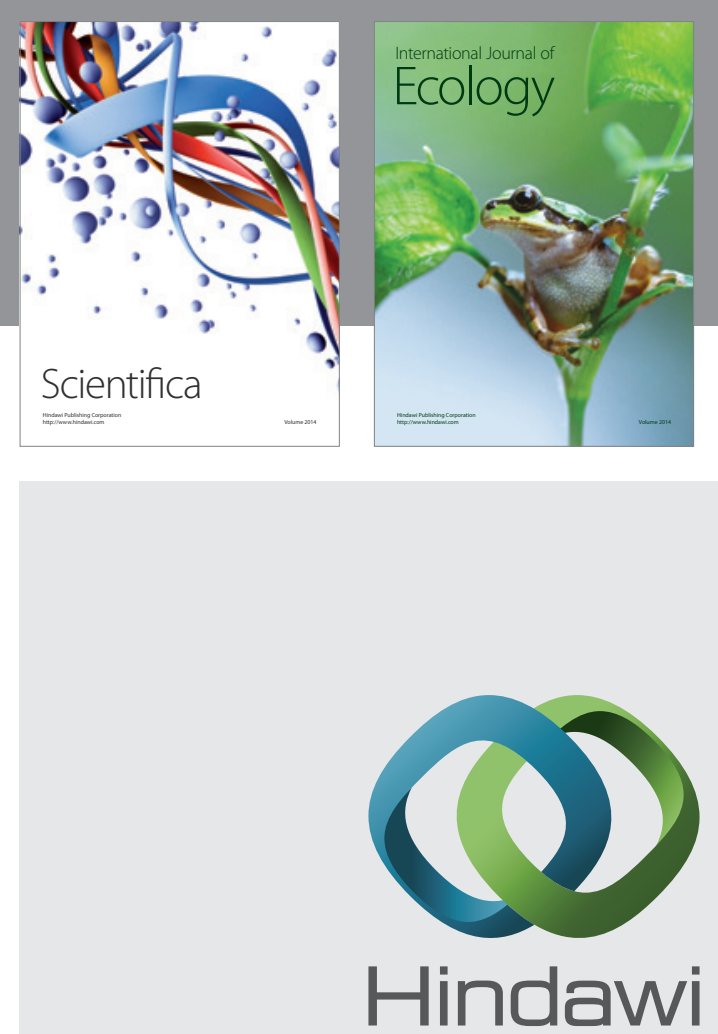

Submit your manuscripts at http://www.hindawi.com
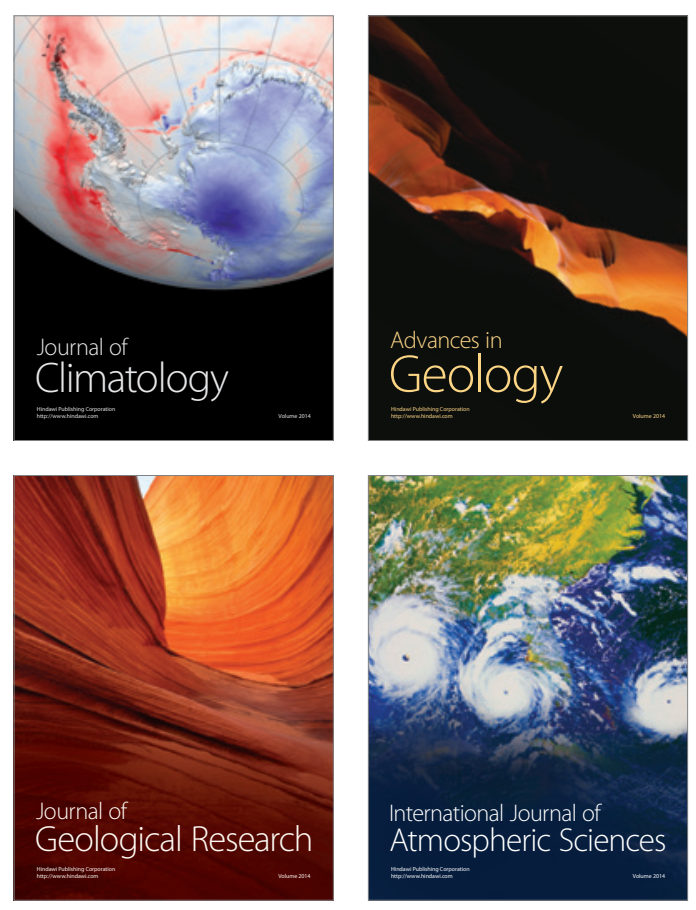
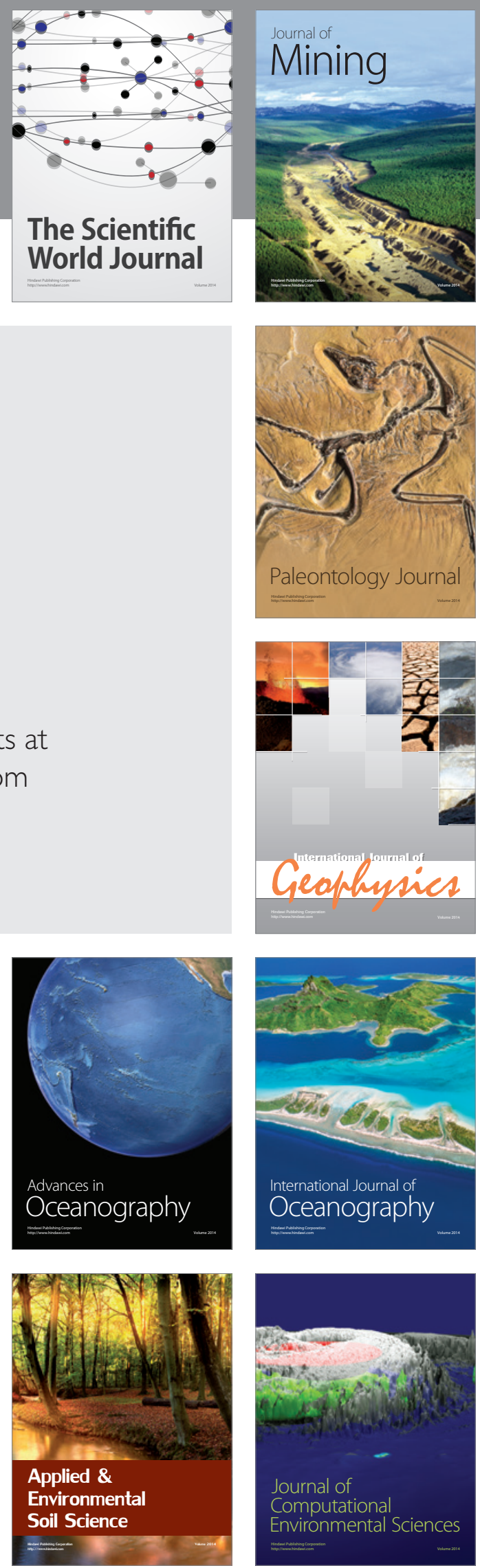